Check for updates

Cite this: Chem. Sci., 2019, 10, 10524

๑ All publication charges for this article have been paid for by the Royal Society of Chemistry

Received 1st August 2019

Accepted 24th September 2019

DOI: $10.1039 / c 9 s c 03829 a$

rsc.li/chemical-science

\section{A hybrid blue perovskite ametal-organic gel (MOG) nanocomposite: simultaneous improvement of luminescence and stability $\uparrow$}

\author{
Samraj Mollick, $\ddagger^{\mathrm{a}}$ Tarak Nath Mandal, $\stackrel{+}{\mathrm{t}}^{\mathrm{a}}$ Atanu Jana, (D) ${ }^{\mathrm{b}}$ Sahel Fajal ${ }^{\mathrm{a}}$ \\ and Sujit K. Ghosh (D) *ac
}

Blue light-emitting hybrid perovskite nanocrystals (NCs) are promising candidates for optoelectronic applications. However, these NCs suffer severely from low photoluminescence quantum yield (PLQY) and inferior stability under working conditions. Herein, we report, for the first time, a simultaneous dramatic improvement in both the luminescence and the stability of hybrid perovskite NCs through embedding in a porous metal-organic gel (MOG) matrix. The nanocomposite (EAPbBr 3 @MOG, EA: ethylammonium) shows sharp emission in the intense blue region $\left(\lambda_{\max }<440 \mathrm{~nm}\right.$ ), with a substantial ten-fold enhancement in the PLQY ( 53\%) compared with $\mathrm{EAPbBr}_{3} \mathrm{NCs}$ (PLQY $~ 5 \%$ ). Incorporation of perovskite NCs into the soft MOG matrix provides the additional benefits of flexibility as well as water stability. As a proof of principle, these nanocomposites were further utilized to fabricate a white lightemitting diode. The combination of high brightness, stability and flexibility of these nanocomposites could render them viable contenders in the development of efficient, blue light-emitting diodes for practical applications

\section{Introduction}

Organic-inorganic hybrid perovskite nanocrystals (NCs) have attracted significant interest in both fundamental and applied research, owing to their spectacular optoelectronic properties. $^{1-3}$ In particular, tunable light emission across the visible region, with high color purity, has seen them be used widely as active materials for lighting and display technology. ${ }^{4-6}$ Recently, a significant amount of research has been concentrated on achieving highly luminescent red and green light-emitting perovskites. $^{7,8}$ Nevertheless, the development of blue lightemitting perovskites, which are essential for full-color display applications, lags significantly behind that of their green and red counterparts. ${ }^{9-12}$ This bottleneck in development is attributed to relatively low defect tolerance, short charge carrier mobility, low photoluminescence quantum yield (PLQY), the existence of detrimental non-radiative trap states, and inferior

\footnotetext{
${ }^{a}$ Department of Chemistry, Indian Institute of Science Education and Research (IISER) Pune, Dr. HomiBhabha Road, Pashan, Pune 411008, India. E-mail: sghosh@gmail. com

${ }^{b}$ Center for Superfunctional Materials, Department of Chemistry, School of Natural Science, Ulsan National Institute of Science and Technology (UNIST), Ulsan 44919, South Korea

${ }^{c}$ Centre for Energy Science, IISER Pune, Pune 411008, India

$\dagger$ Electronic supplementary information (ESI) available: Details of characterization, stability test in detail, and photocatalysis graphs. See DOI: 10.1039/c9sc03829a

\$ These authors contributed equally.
}

stability. ${ }^{\mathbf{1 3 - 1 5}}$ Hence, recent attention has turned towards the development of hybrid blue light-emitting materials, due to their high synthetic feasibility and cost effectiveness. ${ }^{\mathbf{1 6 , 1 7}}$ However, most of them fall into the sky-blue-emitting region, i.e. $480-500 \mathrm{~nm}$, which is not adequate to satisfy the color standard as defined by the National Television System Committee (NTSC) and thus is not competent for a wide-colorgamut display. ${ }^{\mathbf{1 8 , 1 9}}$ Therefore, the development of intense blue $\left(\lambda_{\max }<440 \mathrm{~nm}\right)$ hybrid perovskites is almost indispensable in order to meet the NTSC color standard. The production of intense blue perovskite, however, remains extremely challenging, especially in terms of luminescence and material stability. ${ }^{\mathbf{2 0 2 1}}$ Furthermore, simultaneous improvement of both the photoluminescence and the stability has been widely explored for inorganic perovskite materials, but such concurrent improvements have still proven to be illusive for hybrid perovskite materials.

Encapsulation strategies have been considered as an efficient approach to enhance this optical property, as well as the chemical stability of perovskite NCs, and porous metal-organic frameworks (MOFs) are the latest addition, in this context. ${ }^{22-25}$ However, the heterogeneous properties of MOFs are one of the key issues that restrict further utilization of perovskite-MOF nanocomposites in the form of thin films or different workable structures. ${ }^{26}$ In light of the growing interest in flexible electronics, perovskite NCs embedded with soft materials could offer high tolerance to structural deformation. ${ }^{27}$ We envisioned that a soft porous metal-organic gel (MOG) could be a suitable 
choice as the hosting matrix, having the dual characteristics of a MOF and a gel in terms of tunable porosity and high specific surface area, along with high crack resistance capability and good foldability to form desired shapes. ${ }^{28}$ Plenty of effort has been devoted towards integration of perovskite NCs into numerous polymers, silica and other crystalline materials, but, so far, the porous MOG matrix has never been used to host perovskite NCs. ${ }^{29-32}$ Therefore, porous MOG would be a new addition as a host matrix for encapsulating perovskite NCs.

Herein, we have used a previously reported $\mathrm{Al}(\mathrm{III})$-based, hydrolytically stable hierarchical porous MOG (pore size varies from $2.5 \mathrm{~nm}$ to $10 \mathrm{~nm}$ ), which is analogous to MIL-100(Al). ${ }^{33}$ This MOG matrix was utilized as a protecting host matrix to stabilize hybrid bromide perovskite NCs (HBP-NCs) from aerial or chemical degradation. We have also demonstrated that, through encapsulation within a MOG matrix, weakly blue lightemitting, two-dimensional $\mathrm{EAPbBr}_{3} \mathrm{NCs}^{34,35}$ (PLQY $\sim 5 \%$ ) have achieved an unprecedented ten-fold enhancement in PLQY $(\sim 53 \%)$ without altering the PL peak position. It is noteworthy that, for the first time, simultaneous improvement of both PLQY and water stability has been achieved for intense blueemitting hybrid perovskite NCs. These materials maintained their basic structures as well as their photoluminescence behaviors, even after immersing in water or following UV light irradiation for prolonged periods of time. Furthermore, flexible HBP@MOG nanocomposites were easily molded into various artificial three-dimensional sculptures by virtue of the soft nature of the nanocomposites. To validate this proof of concept, blue emissive $\mathrm{EAPbBr}_{3} @ \mathrm{MOG}$ was selected to fabricate a white light-emitting diode (WLED), by combining it with green and red light-emitting materials on a UV LED chip. The asfabricated device exhibited a wide-color-gamut (144\% of NTSC standard) and showed its potential for application in display technologies.

\section{Experimental}

\section{Materials}

Lead(II) bromide $\left(\mathrm{PbBr}_{2}, 99.999 \%\right)$, hydrobromic acid ( $\mathrm{HBr}$, $48 \%), \mathrm{Al}\left(\mathrm{NO}_{3}\right)_{3} \cdot 9 \mathrm{H}_{2} \mathrm{O}(99.997 \%)$, and trimesic acid (98\%) were purchased from Sigma-Aldrich. Methylamine (40\%), ethylamine $(70 \%)$, and $n$-octylamine (99\%) were purchased from Spectrochem. All solvents were purchased from Finer India and all were used without further purification. Ethylammonium bromide $\left(\mathrm{CH}_{3} \mathrm{CH}_{2} \mathrm{NH}_{3} \mathrm{Br}, \mathrm{EABr}\right)$, methylammonium bromide $\left(\mathrm{CH}_{3} \mathrm{NH}_{3} \mathrm{Br}, \mathrm{MABr}\right)$, and $n$-octylammonium bromide $\left(\mathrm{CH}_{3}(-\right.$ $\left.\mathrm{CH}_{2}\right)_{7} \mathrm{NH}_{3} \mathrm{Br}$, OABr) were synthesized by a previously reported literature method. ${ }^{36}$ A $1 \mathrm{~W}$ UV LED (wavelength $\sim 380 \mathrm{~nm}$ ) was used for fabrication of the white light-emitting LED. Pristine metal-organic gel (MOG) was synthesized following the protocol reported in the literature. ${ }^{33}$

\section{Synthesis of $\mathrm{EAPBBr}_{3} @ \mathrm{MOG}$}

In a typical synthesis protocol, first, $105 \mathrm{mg}$ of trimesic acid (0.5 $\mathrm{mmol}$ ) was dissolved in $14 \mathrm{ml}$ of dimethylformamide (DMF) in a drum vial followed by addition of $281.4 \mathrm{mg}$ of $\mathrm{Al}\left(\mathrm{NO}_{3}\right)_{3} \cdot 9 \mathrm{H}_{2} \mathrm{O}$
$(0.75 \mathrm{mmol})$ to the solution mixture. Next, $70 \mu \mathrm{L}$ of triethylamine $(0.5 \mathrm{mmol})$ was added to the reaction mixture and sonicated to form a clear solution. After that $183 \mathrm{mg}$ of $\mathrm{PbBr}_{2}$ $(0.5 \mathrm{mmol}), 63 \mathrm{mg}$ of EABr $(0.5 \mathrm{mmol})$ and $63 \mathrm{mg}$ of $\mathrm{OABr}(0.3$ $\mathrm{mmol}$ ) were added to the whole reaction mixture and again sonicated to form a clear solution. The whole reaction mixture was kept at $110{ }^{\circ} \mathrm{C}$ for 24 hours and then cooled to room temperature over 10 hours, leading to synthesis of colorless jelly-type metal-organic gel (MOG) materials. During the heating process, MOG was formed in which the perovskite components were trapped inside the MOG matrix. This material was dried at $120^{\circ} \mathrm{C}$ for 2 hours leading to formation of a yellowishcolored xerogel. This xerogel was subjected to stirring for the next 24 hours in the presence of $30 \mathrm{~mL}$ toluene (nucleating solvent) to obtain the $\mathrm{EAPbBr}_{3} @ \mathrm{MOG}$ composite material. Last, the resulting composite was collected by filtration and dried under vacuum overnight to obtain the final product. The $\mathrm{MAPbBr}_{3} @ \mathrm{MOG}$ composite was also prepared following the same procedure, where MABr was used instead of EABr keeping all other components and their amounts same.

\section{Red-emitting Mn(II)-doped EAPbBr}

The red-emitting $\mathrm{Mn}$ (II)-doped $\mathrm{EAPbBr}_{3}$ was prepared following the protocol reported in the literature. ${ }^{34}$ In a typical synthesis, $36.7 \mathrm{mg}$ of $\mathrm{PbBr}_{2}(0.1 \mathrm{mmol}), 11.2 \mathrm{mg}$ of EABr $(0.1 \mathrm{mmol})$ and $12.6 \mathrm{mg}$ of $\mathrm{OABr}(0.06 \mathrm{mmol})$ were ground with a pestle and mortar at room temperature for $30 \mathrm{~min}$. Next, $4.3 \mathrm{mg}$ of $\mathrm{MnBr}_{2}$ $(0.02 \mathrm{mmol})$ was added and ground for another $30 \mathrm{~min}$. After that, $3 \mathrm{ml}$ acetone was added and the mixture was centrifuged at $5000 \mathrm{rpm}$ for $2 \mathrm{~min}$. The precipitate was collected and dried in vacuum for further use as a red light-emitting material.

\section{Water stability}

The water stability tests were carried out in a $5 \mathrm{ml}$ glass vial. Each glass vial contained $6 \mathrm{mg}$ of composite material and was immersed in $2.5 \mathrm{ml}$ water in the open air for 12 hours. During this period of time, the photoluminescence (PL) spectra were examined regularly in the dispersed phase.

\section{Photostability}

Photostability tests were performed in the dark in a $5 \mathrm{ml}$ glass vial. Each glass vial contained $6 \mathrm{mg}$ of composite material and was placed under $365 \mathrm{~nm}$ UV light for 300 hours in the open air. After that, PL emission spectra (dispersed phase in toluene) and powder X-ray diffraction (PXRD) spectra were examined.

\section{Fabrication of thin films}

The pristine composite materials were dispersed in toluene and kept for 3 days, leading to formation of a jelly-type morphology. The obtained jelly-type materials were kept on a glass slide and in the open air for another 7 days for formation of a thin film.

\section{Preparation of various sculptures}

A variety of desired geometrical shapes and different-sized sculptures were prepared using pristine composite materials. 
First, pristine composite materials were dispersed in a minimum amount of toluene and sonicated for 2 hours. After sonication, the composite materials were stirred for one day and kept for another 3 days without stirring at room temperature. The obtained jelly-type materials were poured into different shaped molds and kept for another 3 days to obtain the various-shaped sculptures.

\section{Fabrication of WLED}

The proper ratio of blue, green and red light-emitting materials $\left(\mathrm{Mn}\right.$ (II)-doped $\mathrm{EAPbBr}_{3}$ ) were ground thoroughly for 1.5 hours and dispersed in toluene solution. The resulting mixture was sonicated for $5 \mathrm{~min}$ and then coated on a UV LED. Finally, the coated UV LED was kept at room temperature for 2 days to obtain the WLED.

\section{Characterizations and physical measurements}

PXRD patterns were recorded on a Bruker D8 Advanced X-ray diffractometer at room temperature using $\mathrm{CuK} \alpha$ radiation $(\lambda$ $=1.5406 \AA$ ) at a scan speed of $0.5^{\circ} \mathrm{min}^{-1}$ and a step size of $0.01^{\circ}$ in $2 \theta$. The morphology of the crystalline materials was recorded with a Zeiss Ultra Plus field emission scanning electron microscope (FESEM) with an integral charge compensator and embedded EsB and AsB detectors (Oxford X-max Instruments 80 $\mathrm{mm}^{2}$ (Carl Zeiss NTS, GmbH)). The elemental analysis was carried out using a voltage of $15 \mathrm{kV}$ with an energy-dispersive $\mathrm{X}$ ray spectroscopy (EDS) detector. The steady-state photoluminescence studies were recorded on a Fluorolog-3 spectrofluorometer (Horiba Scientific). Absolute PLQY measurements for the composite materials (dispersed in toluene) were recorded using an integrating sphere according to the reported literature method. ${ }^{37} \mathrm{UV}$-vis absorption studies were performed on a Shimadzu UV 3600 UV/vis/NIR spectrophotometer in an optical quartz cuvette (10 $\mathrm{mm}$ path length) over the entire range of 200-800 nm. The infrared (IR) spectra were acquired using a Nicolet 6700 FTIR spectrophotometer using $\mathrm{KBr}$ pellet in the range 400-4000 $\mathrm{cm}^{-1}$. Gas adsorption measurements were performed using a BelSorp-Max instrument (Bel, Japan). Prior to adsorption measurements, the activated samples were heated at $130{ }^{\circ} \mathrm{C}$ under vacuum for 6 hours using a BelPrepvacII. For highresolution transmission electron microscopy (HRTEM) analysis, all the samples were dispersed in toluene $\left(3 \mathrm{mg} \mathrm{mL} \mathrm{m}^{-1}\right)$ and sonicated for $30 \mathrm{~min}$. Then, the samples were left for $2 \mathrm{~min}$ and the upper part of the solution was taken for preparing TEM samples on a lacey, carbon-coated copper grid (Electron Microscopy Science). TEM imaging and scanning TEM-EDS (STEM-EDS) were performed on the HRTEM (JEM-2100F, JEOL) operating at an acceleration voltage of $200 \mathrm{kV}$. X-ray photoelectron spectroscopy (XPS) measurements were performed on the photoelectron spectrometer (Thermo, K-alpha). The PL decay dynamics (time-correlated single photon counting) were measured with an FLS 980 (Edinburgh Instruments) using $405 \mathrm{~nm}$ pulse laser irradiation with a pulse repetition rate of $500 \mathrm{kHz}$. Decay profiles were fitted to multi-exponential decay curves, and the calculations of average lifetimes were measured using the equation $\tau_{\mathrm{avg}}=\frac{\sum A_{i} \tau_{i}{ }^{2}}{\sum A_{i} \tau_{i}}$, where $A_{i}$ and $\tau_{i}$ are the amplitude and lifetime of the $i$ th component, respectively.

\section{Results and discussion}

The hydrolytically stable Al(III)-based porous MOG was synthesized and thoroughly characterized according to the literature report by Fischer and co-workers (Fig. S1-S6†). ${ }^{33}$ EA/ $\mathrm{MAPbBr}_{3} @ \mathrm{MOG}$ nanocomposites (MA denotes methylammonium) were prepared rapidly via a two-step synthetic protocol. In a typical synthesis process, in the first step, all reacting components were mixed in DMF and heated at $110{ }^{\circ} \mathrm{C}$ for 24 hours. During this process, colorless MOG was formed where perovskite precursors were trapped inside the MOG matrix. In the second step, nucleation of the perovskite NCs was initiated with addition of copious amounts of nucleating solvent (toluene) to form the desired nanocomposites (see Experimental section for more details, Scheme 1, Fig. S7 and S8, $\left.\mathrm{ESI}_{\dagger}\right)^{22}$

The structural analyses of the nanocomposites were confirmed by FESEM, PXRD and TEM studies. FESEM images of the $\mathrm{EAPbBr}_{3} @ \mathrm{MOG}$ composite (Fig. S9, ESI $\dagger$ ) showed a distinctly different morphology compared with the bare perovskite NCs, as well as the MOG materials, and evidence for their micro-sized nature. The STEM-EDS analysis (Table S1, $\mathrm{ESI} \dagger$ ) revealed the existence of excess $\mathrm{Br}$ (Table S2, ESI $\dagger$ ) in the $\mathrm{EAPbBr}_{3} @ \mathrm{MOG}$ surface, which also indicated generation of highly emissive perovskite NCs. ${ }^{38}$ Elemental mapping analysis (Fig. S10, ESI $\dagger$ ) showed distribution of $\mathrm{EAPbBr}_{3}$ NCs throughout the composite material. The formation of crystalline HBP-NCs in the nanocomposites was analyzed through PXRD (Fig. S11 and S12, ESI $\dagger$ ) and the appearance of Bragg's diffraction patterns confirmed the encapsulation of nano-sized HBP-NCs

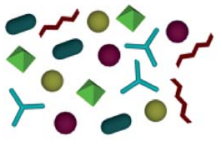

All building units

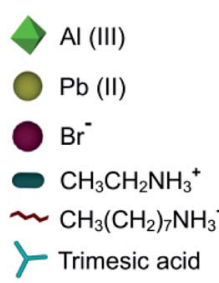

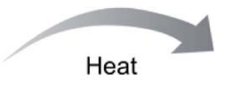

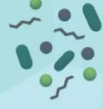

Perovskite components inside MOG

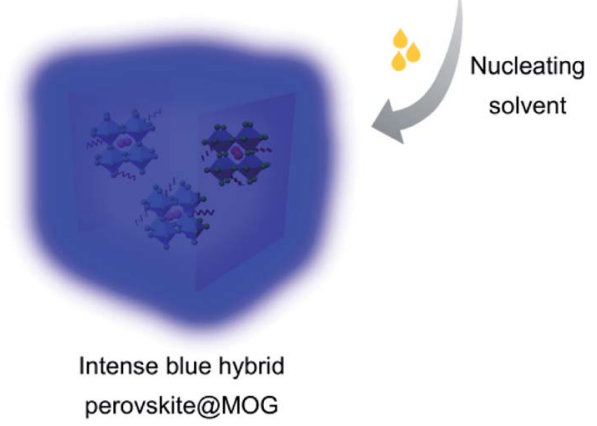

Scheme 1 Schematic of synthetic procedure for synthesis of intense blue hybrid perovskite@MOG nanocomposite. 

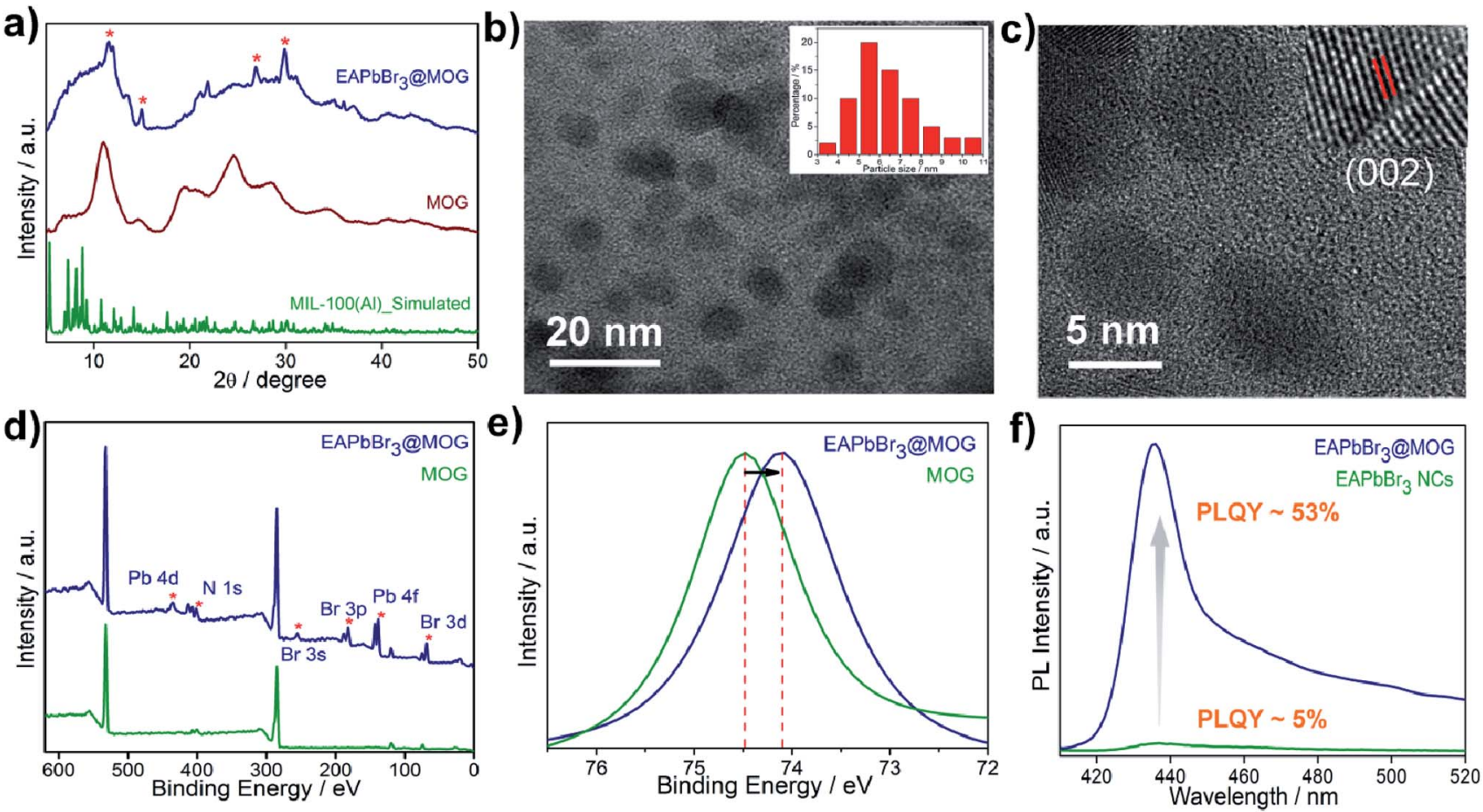

Fig. 1 Characterization of EAPbBr 3 @MOG nanocomposite. (a) PXRD patterns of simulated MIL-100(Al), MOG (pristine) and EAPbBr 3 (MOG nanocomposite. (*) represents the characteristic diffraction peaks for EAPbBr 3 NCs. (b) TEM image of EAPbBr 3 @MOG nanocomposite. Inset: size

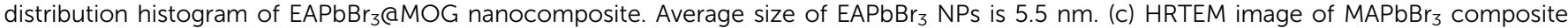
material. Inset: shows the lattice fringe of an individual EAPbBr 3 NC. (d) Full range of XPS spectra of MOG and EAPbBr 3 (aMOG nanocomposite. (*) indicates the characteristic peaks for $\mathrm{EAPbBr}_{3}$ perovskite NCs. (e) XPS spectra of Al $2 p$ for MOG and EAPbBr $\mathrm{CMOG}_{3}$ nanocomposite. (f) PL spectra of $\mathrm{EAPbBr}_{3} \mathrm{NCs}(\mathrm{PLQY} \sim 5 \%$ ) and $\mathrm{EAPbBr} 3 \mathrm{QMOG}$ nanocomposite (PLQY 53\%).

inside the MOG matrix (Fig. 1a). The diffraction peaks at $11.3^{\circ}$, $26.9^{\circ}$ and $30.8^{\circ}$ in the $\mathrm{EAPBBr}_{3} @ M O G$ composite were assigned to the PXRD pattern of $\mathrm{EAPbBr}_{3}$ NCs, and were in accord with literature reports. ${ }^{39}$

The structure of $\mathrm{EAPbBr}_{3}$ has already been reported in the literature and it has a two-dimensional (2D) arrangement. ${ }^{34,35}$ The characteristic diffraction peak at $\sim 11.3^{\circ}$ confirms the $2 \mathrm{D}$ arrangement of the $\mathrm{EAPbBr}_{3}$ NCs. Structural evolutions of the material were studied by TEM analysis and TEM images (Fig. 1b and S13, ESI $\dagger$ ) and confirmed that $\mathrm{EAPbBr}_{3} \mathrm{NCs}$ are spherical in shape and are dispersed inside the MOG matrix. The particle size distribution histogram for $\mathrm{EAPbBr}_{3} @ \mathrm{MOG}$ (inset in Fig. 1b) shows that the spherically shaped $\mathrm{EAPbBr}_{3} \mathrm{NCs}$ have a diameter ranging from 3 to $11 \mathrm{~nm}$, with an average diameter of $5.5 \mathrm{~nm}$. The differently sized NCs are formed in the composite material due to the presence of hierarchical pores in the host MOG matrix..$^{33}$ The HRTEM images of the EAPbBr ${ }_{3} @ M O G$ composite reveal a well-defined lattice spacing according to the crystallographic parameters for $\mathrm{EAPbBr}_{3} \mathrm{NCs}$ (Fig. 1c). In the inset in Fig. 1c, the composite shows an interplanar distance of $0.296 \mathrm{~nm}$, which is attributed to the (002) plane of $\mathrm{EAPbBr}_{3}$ NCs. ${ }^{39}$ Fourier-transform infrared spectroscopy (FTIR) measurements (Fig. S14 and S15, ESI†ं) also confirm the presence of HBP-NCs inside the MOG matrix. Nitrogen sorption isotherms (Fig. S16, ESI $\dagger$ ) show considerably less gas uptake in

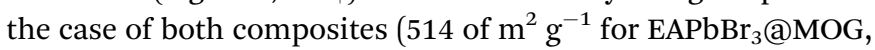

$522 \mathrm{~m}^{2} \mathrm{~g}^{-1}$ for $\mathrm{MAPbBr}_{3} @ \mathrm{MOG}$ ) compared with the parent material, i.e. the MOG $\left(778 \mathrm{~m}^{2} \mathrm{~g}^{-1}\right)$. This suggests that the perovskite NCs are successfully embedded in the MOG matrix. ${ }^{40}$ The full-range XPS spectra of the compounds are shown in Fig. $1 \mathrm{~d}$ and the binding energy positions of different atoms are similar to the literature reports. ${ }^{33,41}$ In the case of the nanocomposites, the appearance of new signals (Fig. S17 and S18, $\mathrm{ESI}+$ ) for bromine and lead atoms proves the formation of HBPNCs in the MOG matrix. Detailed analysis of XPS data (Fig. 1d) show the peak for $\mathrm{Al} 2 \mathrm{p}$ shifted to lower binding energies, from 74.5 to $74.1 \mathrm{eV}$, with a slight peak broadening for the $\mathrm{EAPBBr}_{3} @ \mathrm{MOG}$ compared with the host MOG (Fig. 1e), suggesting a change in the coordination environment around the $\mathrm{Al}$ (III) atoms. It is notable that the negative shift of binding energy for $\mathrm{EAPbBr}_{3} @ \mathrm{MOG}$ occurred due to strong interactions between the MOG framework and the perovskite NCs. The absorption and emission peaks of $\mathrm{EAPbBr}_{3} @ \mathrm{MOG}$ appeared at $398 \mathrm{~nm}$ and $436 \mathrm{~nm}$, respectively, while $\mathrm{MAPbBr}_{3} @ \mathrm{MOG}$ showed an absorption peak at $475 \mathrm{~nm}$ and an emission band at $492 \mathrm{~nm}$ (Fig. S19-S25, ESI $\dagger$ ). The long tail originates in the emission spectrum of $\mathrm{EAPBBr}_{3} @ M O G$, with a full width at halfmaximum height (FWHM) of $18.4 \mathrm{~nm}$ (Fig. S19†), due to the presence of different-sized NCs in the composite material. ${ }^{42}$ Previously, it was also reported that low-dimensional lead halide perovskites exhibited broad band emission due to the formation of self-trapped excitons. ${ }^{43,44}$ Due to the large size of the 
ethylammonium cation $\left(\mathrm{EA}^{+}\right)$, there is a possibility of Jahn-Tellerlike octahedral distortion, ${ }^{45}$ which is responsible for generation of self-trapped excitons. In the $\mathrm{EAPbBr}_{3} @ \mathrm{MOG}$ composite, the tail spans $450 \mathrm{~nm}$ to beyond $520 \mathrm{~nm}$ and is attributed to the formation of self-trapped excitons. Similar PL peak position, PL intensity and FWHM of different batches of composites confirmed the reproducibility of these materials by this two-step synthetic methodology (Fig. S26 and S27, ESI $\dagger$ ). The absolute value of PLQY for the $\mathrm{EAPbBr}_{3}$ @MOG nanocomposite was found to be 53\% (Fig. 1f) and it is noteworthy that, so far, this is one of the highest reported PLQY values among ethyl cation-containing blue light-emitting hybrid perovskite materials. ${ }^{15,39,45}$ Time-resolved photoluminescence spectra of perovskite NCs and composites are shown in Fig. S28-S30† and the fitting parameters are listed in Tables S3 and S4. $\dagger$ The decay profiles of both $\mathrm{EAPbBr}_{3}$ NCs and $\mathrm{EAPbBr}_{3} @ \mathrm{MOG}$ composite are fitted to bi-exponential functions, suggesting two different components are associated in the emission spectra. The average lifetime of the $\mathrm{EAPbBr}_{3} @ \mathrm{MOG}$ nanocomposite (3.03 ns) is much longer compared with the $\mathrm{EAPbBr}_{3}$ NCs $(0.70 \mathrm{~ns})$ (Table S3†), implying successful encapsulation of NCs inside the MOG matrix. ${ }^{\mathbf{4 6}}$ The increased lifetimes indicate the elimination of detrimental trap states and that the generated excitons were promoted to a radiative path through surface passivation via the MOG matrix, as a consequence of the uplift of the PLQY value. ${ }^{47}$ The detrimental non-radiative decay rate constant drops $\left(k_{\mathrm{nr}}, 0.16 \mathrm{~ns}^{-1}\right)$ significantly compared with the parent $\mathrm{EAPbBr}_{3} \mathrm{NCs}\left(k_{\mathrm{nr}}, 1.39 \mathrm{~ns}^{-1}\right)$ (Table S3, ESI $\dagger$ ), resulting in the improved emission behavior of the $\mathrm{EAPbBr}_{3}$ @MOG nanocomposite. $^{48}$ Here, the dramatic improvement in the photoluminescence of the perovskite NCs occurred due to supramolecular interactions between the perovskite material and the MOG matrix. In the as-synthesized $\mathrm{EAPbBr}_{3} @ M O G$ composite, we believe that the presence of both $\mathrm{Al}(\mathrm{III})$ from $\mathrm{Al}-\mathrm{MOG}^{\mathbf{4 9}, 50}$ and carboxylate oxygens from trimesic acid ${ }^{51,52}$ largely removes the surface trap states of $\mathrm{EAPbBr}_{3}$ NCs and, consequently, a giant PL enhancement is observed in the composite material.

\section{Stability test}

All the stability-related experiments for the nanocomposites were executed maintaining similar conditions, in which bare NCs were readily prone to decompose. ${ }^{22,53}$ To ensure open-air stability, both composites were kept in the open air for a few months (Fig. 2a, S31 and S32, ESI $\dagger$ ) and retention of the original PL intensity ( $>80 \%$ ) was found, demonstrating their long-term air stability. The inset in Fig. 2a illustrates the color in the solid state and the PL intensity of the $\mathrm{EAPbBr}_{3} @ \mathrm{MOG}$ remained almost the same, even after four months in the open air, thus proving its long-term environmental stability. Water stability experiments were conducted by immersing the composite materials into water and it was found that, even after 12 hours of immersion, the PL spectra remained almost the same, confirming the outstanding water stability (Fig. 2b, S33 and S34, ESI $\dagger$ ). Photostability tests were performed by measuring PL intensity as a function of time under continuous UV light (365 $\mathrm{nm}$ ) illumination at ambient conditions for 300 hours. Retention of PL intensity ( $>80 \%$, Fig. S35, ESI $\dagger$ ) was found, and the
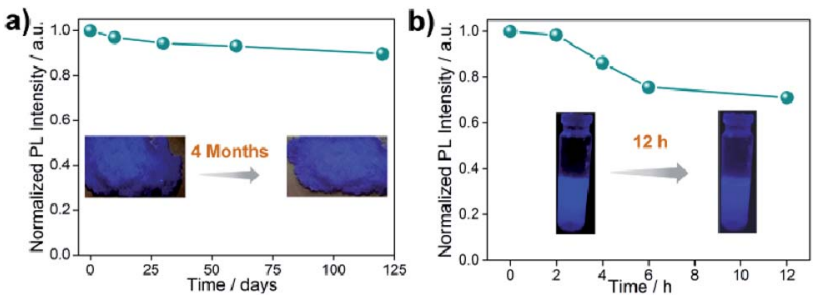

Fig. 2 Stability studies of $\mathrm{EAPbBr}_{3} @ \mathrm{aMOG}$ nanocomposite. (a) Normalized PL intensity as a function of time stored under open air over a period of 4 months. The inset photographs show the color of the solid material under UV light ( $365 \mathrm{~nm}$ ) before and after 4 months. (b) Normalized PL intensity as a function of time in water for different time intervals. Inset photographs show the color of the composites under UV light ( $365 \mathrm{~nm}$ ) before and after 12 hours immersed in water.

PXRD patterns (Fig. S36, ESI $\dagger$ ) of $\mathrm{EAPbBr}_{3} @ \mathrm{MOG}$ remained intact, demonstrating its excellent photostability. The $\mathrm{MAPbBr}_{3} @ \mathrm{MOG}$ composite also showed outstanding stability in the open air, with UV irradiation and even in water media (Fig. S37-S41, ESI $\dagger$ ). In the composite materials, a protecting barrier around the perovskite NCs is formed, which shields the unstable $\mathrm{EAPbBr}_{3} \mathrm{NCs}$ from various kinds of environmental degradation, including water molecules. Because of these shielding effects by the MOG matrix, the water molecules are unable to penetrate the MOG layer and cannot reach the perovskite NCs, leading to excellent stability in open air, water and under UV light. ${ }^{22-24,54}$ Additionally, a variety of well-defined smooth and crack-free artificial shapes and sculptures were successfully fabricated with these composites, without the addition of any external binder (Fig. 3a and S42-S46, ESI†).

Flexible thin films were successfully prepared using these composites for diverse practical applications (Fig. 3a and S47, $\mathrm{ESI} \dagger)$. Table S5 $\dagger$ shows a comparison of the PL properties and stability of the intense blue light-emitting $\left(\lambda_{\max }<440 \mathrm{~nm}\right)$ hybrid perovskites developed so far. This table highlights that the PL properties of our system are comparable to those of the brightest blue light emitters related to hybrid perovskite materials, and with superior stability over other systems.

\section{Fabrication of WLEDs}

As a proof of concept for lighting applications, blue emissive $\mathrm{EAPbBr}_{3} @ \mathrm{MOG}$, green emissive $\mathrm{MAPbBr}_{3} @ \mathrm{MOG}$ and red emissive Mn(II)-doped $\mathrm{EAPbBr}_{3} @ \mathrm{MOG}$ (prepared through mixing $\mathrm{Mn}$ (II) salt with $\mathrm{EAPbBr}_{3}$ NCs) (Fig. S48 and S49, ESI $\dagger$ ) were mixed in the proper ratios and then coupled with a commercially available UV chip to fabricate a WLED device. ${ }^{55,56}$ Fig. $3 \mathrm{~b}$ shows the positions of the emission peak maxima obtained for the WLED, which are in good agreement with the steady-state PL peaks. The photograph of the operating WLED is shown as an inset in Fig. 3b. The Commission Internationale de l'Eclairage (CIE) chromaticity coordinates of the as-prepared WLED were $(0.34,0.32)$ and the color gamut coverage was as high as $144 \%$ (Fig. 3c and S50, ESI $\dagger$ ) of the NTSC standard, benefiting from the narrow emission spectra of the blue and green emissive perovskite composites. The correlated color 
a)
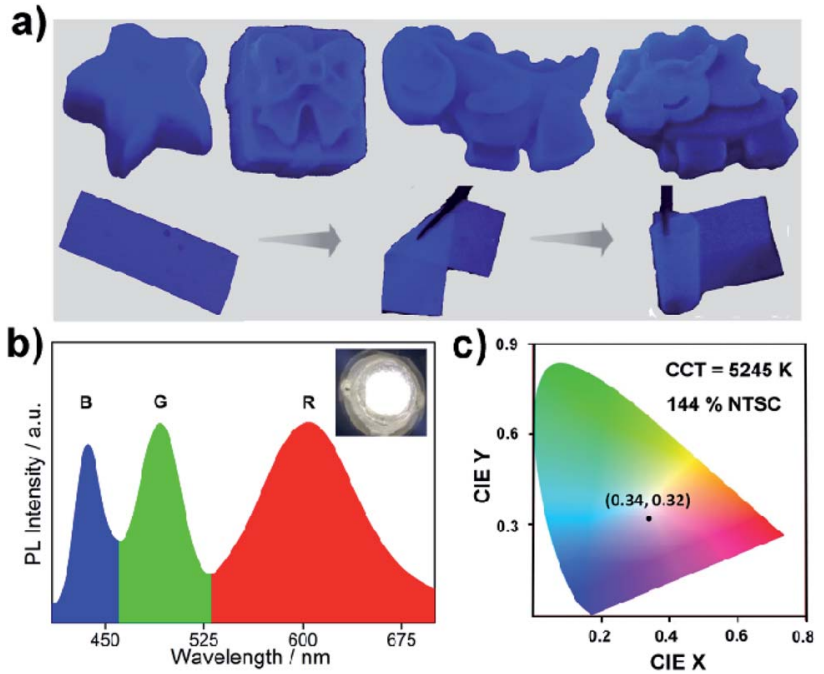

Fig. 3 Different sculptures and characterization of as-fabricated WLEDs. (a) Various sculptures and flexible thin films were fabricated from blue emissive $\mathrm{EAPbBr}_{3} @ M O G$ nanocomposite. The photographs of sculpture and thin film are under UV light (365 nm), showing bright, intense, blue fluorescence. (b) Emission spectrum of white LED fabricated by a combination of blue-emitting $\mathrm{EAPbBr}_{3}(\mathrm{AMOG}(\mathrm{B})$, green-emitting $\mathrm{MAPbBr}_{3} \mathrm{QMOG}(\mathrm{G})$ and red-emitting $\mathrm{Mn}(\|)$-doped $\mathrm{EAPbBr}_{3} \mathrm{NCs}(\mathrm{R})$ deposited on a UV chip. Inset: shows a photograph of the white LED with an applied current of $20 \mathrm{~mA}$. (c) CIE color coordinates of the as-fabricated white LEDs ( $x$ : 0.34, $y$ : 0.32).

temperature (CCT) was $5245 \mathrm{~K}$, which corresponded to so-called white daylight. The WLED maintained its color purity for all components even after 24 hours in the open air, confirming its stability under ambient conditions (Fig. S51, ESI†े).

\section{Conclusions}

In summary, we have developed a straightforward two-step approach for the synthesis of bright, intense, blue light-emitting perovskite@MOG nanocomposites, where perovskite NCs were embedded inside a porous MOG matrix for the first time. Here, the MOG matrix offers dual benefits to the perovskite NCs; namely, it serves as a protective barrier against degradation under working conditions and it enhances the luminescence properties dramatically. Additionally, the flexible nanocomposites were easily fabricated into different, desirable three-dimensional artificial structures for practical use. Finally, the as-synthesized nanocomposites were shown to be applicable as color converters for constructing WLEDs. The present study elucidates the simple fabrication of a cheap, high-quality perovskite-based blue light emitter for future display applications.

\section{Conflicts of interest}

There are no conflicts to declare.

\section{Acknowledgements}

S. M. is thankful to U.G.C. for a research fellowship. T. N. M. thanks SERB (SB/FT/CS-116/2014) for financial support. S. F. acknowledges the DST-Inspire fellowship (DST/INSPIRE/03/ 2016/001694). S. K. G. acknowledges SERB (Project No. EMR/ 2016/000410) and DST Nanomission Thematic Unit for funding.

\section{Notes and references}

1 C. C. Stoumpos and M. G. Kanatzidis, Acc. Chem. Res., 2015, 48, 2791-2802.

2 J. Heo, S. H. Im, J. H. Noh, T. N. Mandal, C.-S. Lim, J. A. Chang, Y. Lee, H.-J. Kim, A. Sarkar, M. K. Nazeeruddin, M. Grätzel and S. I. Seok, Nat. Photonics, 2013, 7, 486-491.

3 J. S. Manser, J. A. Christians and P. V. Kamat, Chem. Rev., 2016, 116, 12956-13008.

4 M. M. Lee, J. Teuscher, T. Miyasaka, T. N. Murakami and H. J. Snaith, Science, 2012, 338, 643-647.

5 L. Protesescu, S. Yakunin, M. I. Bodnarchuk, F. Krieg, R. Caputo, C. H. Hendon, R. X. Yang, A. Walsh and M. V. Kovalenko, Nano Lett., 2015, 15, 3692-3696.

6 Z.-K. Tan, R. S. Moghaddam, M. L. Lai, P. Docampo, R. Higler, F. Deschler, M. Price, A. Sadhanala, L. M. Pazos, D. Credgington, F. Hanusch, T. Bein, H. J. Snaith and R. H. Friend, Nat. Nanotechnol., 2014, 9, 687-692.

7 X. Dai, Y. Deng, X. Peng and Y. Jin, Adv. Mater., 2017, 29, 1607022.

8 M. V. Kovalenko, L. Protesescu and M. I. Bodnarchuk, Science, 2017, 358, 745-750.

9 B. S. Sutherland, Joule, 2018, 2, 2199-2201.

10 B. J. Bohn, Y. Tong, M. Gramlich, M. L. Lai, M. Doblinger, K. Wang, R. L. Z. Hoye, P. Muller-Buschbaum, S. D. Stranks, A. S. Urban, L. Polavarapu and J. Feldmann, Nano Lett., 2018, 18, 5231-5238.

11 W. Liu, W. P. Lusting and J. Li, EnergyChem, 2019, 1, 100008. 12 Z. Wei, Z.-Y. Gu, R. K. Arvapally, Y.-P. Chen, R. N. McDougald Jr, J. F. Ivy, A. A. Yakovenko, D. Feng, M. A. Omary and H.-C. Zhou, J. Am. Chem. Soc., 2014, 136, 8269-8276.

13 G. H. Ahmed, J. K. El-Demellawi, J. Yin, J. Pan, D. B. Velusamy, M. N. Medhili, E. Alarousu, O. M. Bakr, H. N. Alshareef and O. F. Mohammed, ACS Energy Lett., 2018, 3, 2301-2307.

14 Y. Wu, C. Wei, X. Li, Y. Li, S. Qiu, W. Shen, B. Cai, Z. Sun, D. Yang, Z. Deng and H. Zeng, ACS Energy Lett., 2018, 3, 2030-2037.

15 X. Gong, O. Voznyy, A. Jain, W. Liu, R. Sabatini, Z. Piontkowski, G. Walters, G. Bappi, S. Nokhrin, O. Bushuyev, M. Yuan, R. Comin, D. McCamant, S. O. Kelley and E. H. Sargent, Nat. Mater., 2018, 17, 550-556. 16 T. M. Brenner, D. A. Egger, L. Kronik, G. Hodes and D. Cahen, Nat. Rev. Mater., 2016, 1, 15007.

17 Y. Wu, X. Li and H. Zeng, ACS Energy Lett., 2019, 4, 673-681. 18 J. Xing, Y. Zhao, M. Askerka, L. N. Quan, X. Gong, W. Zhao, J. Zhao, H. Tan, G. Long, L. Gao, Z. Yang, O. Voznyy, J. Tang, Z.-H. Lu, Q. Xiong and E. H. Sargent, Nat. Commun., 2018, 9, 3541.

19 Y. Jiang, C. Qin, M. Cui, T. He, K. Liu, Y. Huang, M. Luo, L. Zhang, H. Xu, S. Li, J. Wei, Z. Liu, H. Wang, G.-H. Kim, M. Yuan and J. Chen, Nat. Commun., 2019, 10, 1868. 
20 N. K. Kumawat, X.-K. Liu, D. Kabra and F. Gao, Nanoscale, 2019, 11, 2109-2120.

21 M. K. Gangishetty, S. Hou, Q. Quan and D. N. Conreve, Adv. Mater., 2018, 30, 1706226.

22 S. Mollick, T. N. Mandal, A. Jana, S. Fajal, A. V. Desai and S. K. Ghosh, ACS Appl. Nano Mater., 2019, 2, 1333-1340.

23 H. He, Y. Cui, B. Li, B. Wang, C. Jin, J. Yu, L. Yao, Y. Yang, B. Chen and G. Qian, Adv. Mater., 2019, 31, 1806897.

24 Z. Chen, Z.-G. Gu, W.-Q. Fu, F. Wang and J. Zhang, ACS Appl. Mater. Interfaces, 2016, 8, 28737-28742.

25 X.-Y. Liu, K. Xiang, Y. Li, C.-K. Tsung and J. Li, J. Am. Chem. Soc., 2019, 141, 14807-14813.

26 V. Urbanova, K. Jayaramulu, A. Schneemann, S. Kment, R. A. Fischer and R. Zboril, ACS Appl. Mater. Interfaces, 2018, 10, 41089-41097.

27 X. Wang, X. Lian, Z. Zhang and H. Gao, ACS Energy Lett., 2019, 4, 1446-1454.

28 X. Yang, G. Zhang and D. Zhang, J. Mater. Chem., 2012, 22, 38-50.

29 Y. Yang, J. T. Lee, T. Liyanage and R. Sardar, J. Am. Chem. Soc., 2019, 141, 1526-1536.

30 G. Li, F. W. R. Rivarola, N. J. L. K. Davis, S. Bai, T. C. Jellicoe, F. de la Pena, S. Hou, C. Ducati, F. Gao, R. H. Friend, N. C. Greenham and Z.-K. Tan, Adv. Mater., 2016, 28, 35283534.

31 V. Malgras, S. Tominaka, J. W. Ryan, J. Henzie, T. Takei, K. Ohara and Y. Yamauchi, J. Am. Chem. Soc., 2016, 138, 13874-13881.

32 A. Loiudice, S. Saris, E. Oveisi, D. T. L. Alexander and R. Buonsanti, Angew. Chem., Int. Ed., 2017, 56, 10696-10701.

33 K. Jayaramulu, F. Geyer, M. Petr, R. Zboril, D. Vollmer and R. A. Fischer, Adv. Mater., 2017, 29, 1605307.

34 B. Luo, Y. Guo, X. Li, Y. Xiao, X. Huang and J. Z. Zhang, J. Phys. Chem. C, 2019, 123, 14239-14245.

35 S. Yun, A. Kirakosyan, S.-G. Yoon and J. Choi, ACS Sustainable Chem. Eng., 2018, 6, 3733-3738.

36 G. C. Papavassiliou, G. Pagona, N. Karousis, G. A. Mousdis, I. Koutselas and A. Vassilakopoulou, J. Mater. Chem., 2012, 22, 8271-8280.

37 J. C. de Mello, H. F. Wittmann and R. H. Friend, Adv. Mater., 1997, 9, 230-232.

38 F. Zhang, H. Zhong, C. Chen, X.-G. Wu, X. Hu, H. Huang, J. Han, B. Zou and Y. Dong, ACS Nano, 2015, 9, 4533-4542.

39 Q. Wang, J. Ren, X.-F. Peng, X.-X. Ji and X.-H. Yang, ACS Appl. Mater. Interfaces, 2017, 9, 29901-29906.
40 X. Gu, Z.-H. Lu, H.-L. Jiang, T. Akita and Q. Xu, J. Am. Chem. Soc., 2011, 133, 11822-11825.

41 C. Zhang, B. Wang, W. Li, S. Huang, L. Kong, Z. Li and L. Li, Nat. Commun., 2017, 8, 1138.

42 M. Mittal, A. Jana, S. Sarkar, P. Mahadevan and S. Sapra, J. Phys. Chem. Lett., 2016, 7, 3270-3277.

43 M. D. Smith, B. A. Connor and H. I. Karunadasa, Chem. Rev., 2019, 119, 3104-3139.

44 H. Lin, C. Zhou, J. Neu, Y. Zhou, D. Han, S. Chen, M. Worku, M. Chaaban, S. Lee, E. Berkwits, T. Siegrist, M.-H. Du and B. Ma, Adv. Opt. Mater., 2019, 7, 1801474.

45 D. Cortecchia, J. Yin, A. Petrozza and C. Soci, J. Mater. Chem. C, 2019, 7, 4956-4969.

46 Y. Wang, J. He, H. Chen, J. Chen, R. Zhu, P. Ma, A. Towers, Y. Lin, A. J. Gesquiere, S.-T. Wu and Y. Dong, Adv. Mater., 2016, 28, 10710-10717.

47 Z. Li, L. Kong, S. Huang and L. Li, Angew. Chem., Int. Ed., 2017, 56, 8134-8138.

48 N. Mondal, A. De and A. Samanta, ACS Energy Lett., 2019, 4, 32-39.

49 J. T.-W. Wang, Z. Wang, S. Pathak, W. Zhang, D. W. deQuilettes, F. Wisnivesky-Rocca-Rivarola, J. Huang, P. K. Nayak, J. B. Patel, H. A. M. Yusof, Y. Vaynzof, R. Zhu, I. Ramirez, J. Zhang, C. Ducati, C. Grovenor, M. B. Johnston, D. S. Ginger, R. J. Nicholas and H. J. Snaith, Energy Environ. Sci., 2016, 9, 2892-2901.

50 T. Kyomen, R. Sakamoto, N. Sakamoto, S. Kunugi and M. Itoh, Chem. Mater., 2005, 17, 3200-3204.

51 D. Lu, Y. Zhang, M. Lai, A. Lee, C. Xie, T. Lei, Z. Lin, C. S. Kley, J. Huang, E. Rabani and P. Yang, Nano Lett., 2018, 18, 6967-6973.

52 Y. Tian, M. Peter, E. Unger, M. Abdellah, K. Zheng, T. Pullerits, A. Yartsev, V. Sundstron and I. G. Scheblykin, Phys. Chem. Chem. Phys., 2015, 17, 24978-24987.

53 H. Sun, Z. Yang, M. Wei, W. Sun, X. Li, S. Ye, Y. Zhao, H. Tan, E. L. Kynaston, T. B. Schon, H. Yan, Z.-H. Lu, G. A. Ozin, E. H. Sargent and D. S. Seferos, Adv. Mater., 2017, 29, 1701153-1701162.

54 D. Zhang, Y. Xu, Q. Liu and Z. Xia, Inorg. Chem., 2018, 57, 4613-4619.

55 D. Chen, J. Li, X. Chen, J. Chen and J. Zhong, ACS Appl. Mater. Interfaces, 2019, 11, 10059-10067.

56 M. Bidikoudi, E. Fresta and R. D. Costa, Chem. Commun., 2018, 54, 8150-8169. 